\title{
Baicalein inhibits osteosarcoma cell proliferation and invasion through the miR-183/Ezrin pathway
}

\author{
JIAN ZHANG ${ }^{1}$, WEI YANG ${ }^{1}$, YOU-BING ZHOU ${ }^{1}$, YONG-XIAO XIANG ${ }^{1}$, LU-SHAN WANG ${ }^{2}$, \\ WEN-KAI HU ${ }^{2}$ and WEN-JUN WANG ${ }^{2}$ \\ Departments of ${ }^{1}$ Hand Microsurgery and ${ }^{2}$ Spine Surgery, The First Affiliated Hospital, \\ University of South China, Hengyang, Hunan 421001, P.R. China
}

Received November 27, 2017; Accepted May 9, 2018

DOI: $10.3892 / \mathrm{mmr} .2018 .9036$

\begin{abstract}
Osteosarcoma (OS), a common and primary malignant bone tumor, is characterized by highly aggressive potency. Baicalein, a bioactive flavone isolated from Scutellaria baicalensis Georgi, has been shown to inhibit the progression of numerous tumors, including OS. However, the mechanisms by which baicalein protects against OS are still largely unknown. The results of the present study showed that administration of baicalein significantly inhibited the proliferation, migration and invasion and promoted apoptosis in MG-63 and Saos-2 cells. Ezrin was identified as a target gene of microRNA (miR)-183. MG-63 and Saos-2 cells treated with baicalein exhibited increased miR-183 levels and decreased Ezrin expression. Importantly, miR-183 inhibition and Ezrin overexpression abolished the effects of baicalein on MG-63 and Saos-2 cell proliferation, migration, invasion and apoptosis. Taken together, these findings suggest that baicalein inhibits the proliferation, migration and invasion and induces apoptosis in OS cells by activating the miR-183/Ezrin pathway, revealing a novel mechanism underlying anti-OS effects of baicalein.
\end{abstract}

\section{Introduction}

Osteosarcoma (OS) is regarded as the most common primary malignant bone tumor. It occurs chiefly in children and adolescents who account for $\sim 70 \%$ of all cases (1). Like the vast majority of malignant tumors, OS is highly invasive and has distant metastatic potential, especially in the lung (2). Despite

Correspondence to: Professor Wen-Jun Wang, Department of Spine Surgery, The First Affiliated Hospital, University of South China, 69 Chuanshan Road, Hengyang, Hunan 421001, P.R. China

E-mail: wwwwjj167@qq.com

Abbreviations: OS, Osteosarcoma; SB, Scutellaria baicalensis; miRNAs, microRNAs; 3'-UTR, 3'-untranslated region

Key words: baicalein, miR-183, Ezrin, osteosarcoma surgery in combination with chemotherapy and/or radiotherapy has greatly improved the prognosis of OS patients, five-year survival rate is still lower. Moreover, chemotherapy can lead to drug-resistance and produce life-threatening side effects, such as cardiotoxicity and nephrotoxicity. Thus, there is an urgent need to develop safer and more effective anti-OS drugs.

The biologically active compounds extracted from plants and other natural sources have become an attractive strategy for treating cancers, including OS. Scutellaria baicalensis (SB) Georgi, a traditional Asian herb, is widely applied in the clearance of heat dampness and purgation of fire detoxification (3). Baicalein (5,6,7-trihydroxyflavone), one of the primary active elements of this plant, exerts a variety of pharmacological functions, such as anti-oxidation, anti-proliferation, and apoptosis induction $(4,5)$. There is growing evidence that administration of baicalein inhibits the development of many tumors, including cervical cancer, colorectal cancer, bladder cancer and breast cancer (6-9). Notably, baicalein has also a potent anti-OS effect. However, the potential molecular mechanisms of baicalein on human OS are still vague.

MicroRNAs (miRNAs) are a class of small and non-coding RNA molecules, which can regulate gene expression at the post-transcriptional level by binding to the 3'-untranslated region (UTR) of target mRNAs. Dysregulation of miRNAs is involved in the maligantly biological behaviors of tumors $(10,11)$. As an evolutionarily conserved miRNA, miR-183 has been showed to serve as either a tumor suppressor or oncogene in various human tumors (12). Ezrin gene is located in 6q25 and composed of 585 amino acids and can mediate the tumor invasion and metastasis $(13,14)$. Research has revealed that miR-183 level was dramatically decreased in OS cells and tissues, enhancing the migration and invasion of OS by targeting Ezrin (15). Moreover, our previous study also confirmed that Ezrin aggravated the aggressiveness of OS, and was a direct target of miR-183 (16). Therefore, it could be ascertained that the miR-183/Ezrin pathway plays vital roles in the development and progression of OS. However, there have not yet been any investigations concerning whether the antitumor effects of baicalein on OS were triggered by miR-183/Ezrin pathway until now.

In the present study, we found that baicalein inhibited the proliferation, migration and invasion and induced apoptosis in two human OS cell lines, MG-63 and Saos-2. Importantly, 
activation of the miR-183/Ezrin pathway was required for these effects. These results provide a novel insight into the mechanisms by which baicalein protects against OS.

\section{Materials and methods}

Drugs, reagents and antibodies. Baicalein was obtained from Sigma-Aldrich Chemical Co. (St. Louis, MO, USA). Dulbecco's modified Eagle's medium (DMEM), penicillin/streptomycin, $0.25 \%$ trypsin-EDTA, and dimethyl sulphoxide (DMSO) were purchased from Gibco (Grand Island, NY, USA). Anti-Ezrin (1:500) and anti-phosphor-Ezrin (1:500) antibodies were acquired from Abcam (Cambridge, MA, USA). In addition, anti-GAPDH $(1: 1,000)$ and secondary antibodies $(1: 3,000)$ were obtained from the Cell Signaling Technology (CST; Beverly, MA, USA).

Cell culture. Human OS cell lines MG-63 and Saos-2 were purchased from China Center for Type Culture Collection (Wuhan, China), and human osteoblast cell line hFOB1.19 was obtained from the Chinese Cell Bank of the Chinese Academy of Sciences (Shanghai, China). MG-63 and Saos-2 cells were maintained in complete medium containing DMEM, $10 \%$ fetal bovine serum (FBS; Biolnd, Israel), $50 \mathrm{U} / \mathrm{ml}$ penicillin/streptomycin in a humidified incubator with $5 \% \mathrm{CO}_{2}$ at $37^{\circ} \mathrm{C}$. hFOB1.19 cells were cultured in complete medium consisting of DMEM/F12 (1:1) medium, 10\% FBS (Biolnd, Israel), and $\mathrm{G} 418(0.3 \mathrm{mg} / \mathrm{ml})$ with $5 \% \mathrm{CO}_{2}$ at $34^{\circ} \mathrm{C}$. Sebsequently, these cells were digested by $0.25 \%$ trypsin-EDTA solution for all in vitro experiments and for cell pass and 27age unless stated otherwise after cells reached at least $80 \%$ confluence.

Cell proliferation assay. Baicalein was dissolved in DMSO solution to a final concentration of 4 mM. MG-63, Saos-2 and hFOB cells were seeded at density 5,000 cells/well in 96-well plates and cultured for $24 \mathrm{~h}$. Cells were treated with baicalein at different concentrations $(0,25,50,75$ and $100 \mu \mathrm{M})$ at $0 \mathrm{~h}$, and then were cultured for 24,48 or $72 \mathrm{~h}$. Subsequently, the proliferation assay was performed using a Cell Counting kit- 8 (CCK-8; Dojindo Molecular Technologies, Inc., Kumamoto, Japan) according to the manufacturer's instructions. A microplate reader (Thermolex Molecular Device Co.) was utilized to detect the absorbance at $450 \mathrm{~nm}$.

Cell apoptosis analysis. Following maintenance in culture by different treatments, cell apoptosis was assessed using an Annexin V/fluorescein isothiocyanate (FITC) Apoptosis Detection kit I (BD Biosciences, San Jose, CA, USA). The untreated or treated MG-63 and Saos-2 cells were harvested after indicated treatments with $0.25 \%$ trypsin-EDTA, and a single cell suspension was prepared. Cells were washed twice with cold PBS, centrifugated at $1,500 \mathrm{rpm}$ for $5 \mathrm{~min}$ and resuspended in $1 \mathrm{X}$ Binding Buffer so that cell density reached $1 \times 10^{6}$ cells $/ \mathrm{ml}$. $5 \mu \mathrm{l}$ Annexin V/FITC was added to $300 \mu \mathrm{l}$ the above resuspended solution and incubated for $10 \mathrm{~min}$, followed by adding $5 \mu \mathrm{l}$ propidium iodide (PI) for a 10 min incubation at room temperature in the dark. Cell apoptosis were then detected using flow cytometry (BD Biosciences). The results were analyzed by cell quest software (BD Biosciences) to determine the rate of apoptosis in the lower right quadrant.
Cell invasion and migration assay. Cell invasion assay was carried out using Millicell Hanging Cell Culture Insert (24-well, pore size $5 \mu \mathrm{m}$; Merck Millipore, Billerica, MA, USA). After treatment, MG-63 and Saos-2 cells resuspended to a density of $5 \times 10^{4}$ cells $/ \mathrm{ml}$ in serum-free DMEM medium were transferred into upper chamber of the inserts coated with $30 \mathrm{mg} / \mathrm{cm}^{2}$ of Matrigel (BD Biosciences), DMEM medium with $20 \%$ FBS were added to the well out of the insert and then incubation for different concentration or time based on experimental program. Ultimately, cells on the upper membrane surface were removed through careful wiped off a cotton swab, and the cells on lower surface were fixed with $95 \%$ ethanol for 30 min and stained with $0.2 \%$ Crystal Violet solution for $30 \mathrm{~min}$. Five vision fields per chamber were randomly selected and then counted the number of invasion cells under an inverted microscope. The general procedure of the migration assay was performed the same as that of the invasion assay described above, except that Matrigel was not applied.

RNA extraction and reverse transcription-quantitative polymerase chain reaction $(R T-q P C R)$ analysis. Total RNAs were extracted from either MG-63 or Saos-2 cells using TRIzol reagent (Invitrogen; Thermo Fisher Scientific, Inc., Waltham, MA, USA) according to the manufacturers' protocol. The purity and concentration of RNAs were detected by a NanoDrop ND-1,000 spectrophotometer (Thermo Fisher Scientific, Inc., Wilmington, DE, USA). The reverse transcription reactions were conducted by a miRcute miRNA Reverse Transcription kit (TianGen Biotech Co., Ltd., Beijing, China) and PrimeScript RT Master Mix (Perfect Real Time; Takara Biotechnology Co., Ltd., Dalian, China). All reactions were performed using a 7500 Real-Time PCR System (Thermo Fisher Scientific, Inc.) in a reaction volume of $20 \mu 1$. The levels of miR-183 and Ezrin were normalized to U6 small nuclear RNA and GAPDH, respectively. The relative quantification was calculated by applying the $2^{-\Delta \Delta C \mathrm{q}}$ method (17). The PCR primers were designed and synthesized by Shanghai Sangon Biological Engineering Technology Co., Ltd., (Shanghai, China) (Table I).

Western blot analysis. Total proteins were extracted from MG-63 or Saos-2 cells using RIPA Lysis Buffer (Beyotime, Shanghai, China). The protein concentrations were measured via Enhanced BCA Protein Assay kit (Bio-Rad, Richmond, CA, USA). Lysates of Total protein were separated by a $10 \%$ sodium dodecyl sulfate-polyacrylamide (SDS-PAGE) gel and transferred to a polyvinylidene fluoride (PVDF) membrane (Merck Millipore). The membrane was blocked by $5 \%(\mathrm{v} / \mathrm{v})$ skim milk in Tris-buffered saline Tween-20 (TBST) at room temperature for $2 \mathrm{~h}$, followed by incubation with the primary antibodies at $4^{\circ} \mathrm{C}$ overnight. After washing, the membrane was incubated with HRP-conjugated secondary antibody at room temperature for $1 \mathrm{~h}$. The proteins were visualized using a chemiluminescence method (electrochemiluminescence and western blot detection system; Amersham Biosciences, Foster City, CA, USA). The results were normalized using GAPDH.

Cell transfection. MiR-183 mimics/negative controls and inhibitors/negative controls were supplied by 
Table I. Primer sequences used for RT-qPCR analyses.

\begin{tabular}{lc}
\hline Gene & Sequence \\
\hline miR-183 & \\
Forward & 5'-TATGGCACTGGTAGAATTCACT-3' \\
Reverse & 5'-GCGAGCACAGAATTAATACGAC-3' \\
U6 & \\
Forward & 5'-CGCTTCGGCAGCACATATAC-3' \\
Reverse & 5'-AAATATGGAACGCTTCACGA-3' \\
Ezrin & \\
Forward & 5'-TGGGATGCTCAAAGATAATGC-3' \\
Reverse & 5'-ACTCCAAGCCAAAGGTCTGTT-3' \\
GAPDH & \\
Forward & 5'-GGCACAGTCAAGGCTGAGAATG-3' \\
Reverse & 5'-ATGGTGGTGAAGACGCCAGTA-3'
\end{tabular}

miR, microRNA; RT-qPCR, reverse transcription-quantitative polymerase chain reaction.

RiboBio (Guangzhou, China). The whole DNA fragment of Ezrin was amplified from the OS cells. The obtained products were cloned into recombinant plasmid pcDNA3.1(+)-EGFP to overexpress Ezrin (pcDNA3.1-Ezrin; Invitrogen; Thermo Fisher Scientific, Inc.), with the empty vector as a negative control (pcDNA3.1-Mock). The constructs were identified by DNA sequencing. Both MG-63 and Saos-2 cells were seeded into a 12 -well plate at a $1 \times 10^{5} / \mathrm{ml}$ density, and then transfected with $20 \mathrm{nM}$ miR-183 mimic/negative controls or $40 \mathrm{nM}$ miR-183 inhibitors/negative controls or pcDNA3.1-Ezrin using Lipofectamine 2000 (Invitrogen; Thermo Fisher Scientific, Inc.) according to the manufacturer's instructions.

Dual luciferase reporter assay. A fragment of 3'UTR of Ezrin containing the putative miR-183 binding site was amplified by RT-PCR. The amplified product was then subcloned into a pMIR-REPORT luciferase reporter vector applying restriction sites XhoI (Promega, Madison, WI, USA). The mutated sequences of predicred miR-183 binding located in the 3'UTR of Ezrin mRNA were introduced using the QuikChange kit (Stratagene, La Jolla, CA, USA). All plasmid constructs were verified by DNA sequencing (Biology Engineering Corp., Shanghai, China). Successively, the $20 \mathrm{nM}$ miR-183 mimics/negative controls or $40 \mathrm{nM}$ miR-183 inhibitor/negative controls and $0.1 \mu \mathrm{g}$ pMIR-REPORT-Ezrin (WT) or pMIR-REPORT-Ezrin (MUT) constructs were co-transfected into the cells using Lipofectamine 2000. After transfection for $48 \mathrm{~h}$, the Dual-Luciferase Reporter Assay System (Promega) was used to detect the luciferase activity. The Renilla luciferase values were regarded as a correction factor, and the firefly/Renilla ratio was obtained. Luciferase activity was averaged from three replicates for each transfection.

Statistical analysis. All data were presented as the means \pm standard deviation (SD), and all of graphs were produced by GraphPad Prism 5.0 Software (Graph Pad Software, Inc., La Jolla, CA, USA). SPSS 22.0 software
(IBM Corp., Armonk, NY, USA) was used for all statistical analyses. Results were analyzed using Student's t-test for comparison between two groups or one-way ANOVA followed by the Tukey's test for comparison of multiple groups. $\mathrm{P}<0.05$ was considered to indicate a statistically significant difference.

\section{Results}

Baicalein inhibits proliferation and promotes apoptosis in MG-63 and Saos-2 cells. To investigate the effect of baicalein on OS cell proliferation, cells were treated with various concentrations of baicalein for the indicated time, followed by the CCK- 8 assay. Our results showed that baicalein inhibited the proliferation of MG-63 and Saos-2 cells in a concentrationand time-dependent manner (Fig. 1A and B), but did not affect hFOB cell growth (Fig. 1C). Given that $100 \mu \mathrm{M}$ baicalein had the optimal efficacy, we selected this dose for subsequent experiments.

Given the fact that the inhibition of apoptosis contributed to cell proliferation in various type of tumor cells, next we employed fluorescence activated cell sorter (FACS) analysis via Annexin V-FITC and PI double-staining to evaluate the apoptosis rate of MG-63 and Saos-2 cells exposed to $100 \mu$ M baicalein for $48 \mathrm{~h}$. As shown in Fig. 1D, the apoptosis rate of MG-63 and Saos-2 cells was low in control group but significantly elevated in baicalein-treated group, suggesting an inductive effect of baicalein on MG-63 and Saos-2 cell apoptosis.

Baicalein represses the migration and invasion of OS cells. It is well known that migration and invasion are the two pivotal stages in malignant progression and metastasis for tumors. Thus, the present study further evaluated the effects of baicalein on the aggressive potential of MG-63 and Saos- 2 cells using cell migration and invasion assays. After stimulation of MG-63 and Saos-2 cells with $100 \mu \mathrm{M}$ baicalein for $48 \mathrm{~h}$, the migration ability of cells was measured. In Fig. 2A, we found that the migration ability of MG-63 and Saos- 2 cells was obviously inhibited. Aside from migration test, we also employed the cell invasion assay to measure the invasion ability of MG-63 and Saos-2 cells. As shown in Fig. 2B, the similar effect on invasive ability was also observed in parallel invasive test, compared with the control group. Altogether, the above results reveal that baicalein can suppress the migration and invasion ability of MG-63 and Saos-2 cells.

Identification of Ezrin as a direct target of $m i R$-183. It has been reported that miRNAs are important regulators in the aggressive behavior of tumor, including OS (18). Our previous study has been proposed that Ezrin as a direct target of miR-183 promotes the migration and invasion of OS MG-63 cells through increased $\mathrm{N}$-cadherin and activating ERK signaling (16). However, whether baicalein regulates the activation of miR-183/Ezrin pathway in MG-63 and Saos-2 cells remains unclear. In order to explore this hypothesis, we validated again the relationship between Ezrin and miR-183 in the current research. Treatment of MG-63 and Saos-2 cells with miR-183 mimic/negative control or miR-183 inhibitors/negative control both exerted a higher transfection efficiency (Fig. 3A). The pairing site of Ezrin 3'-UTR with miR-183 were predicted by TargetScan (an open sourced software) (Fig. 3B). A 


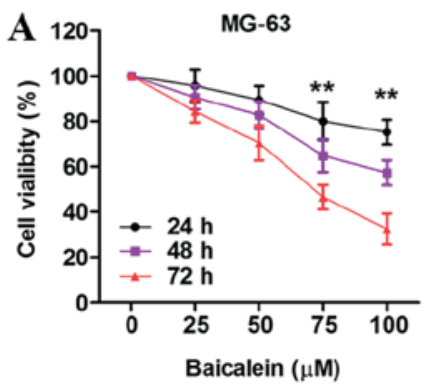

D

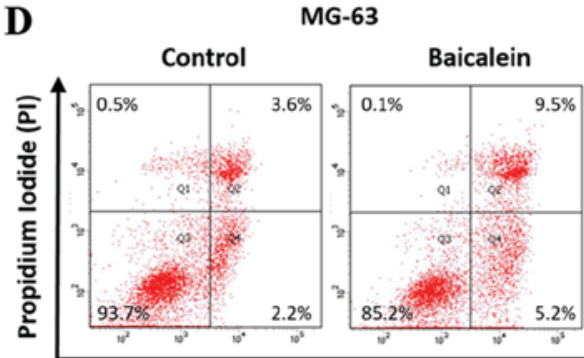

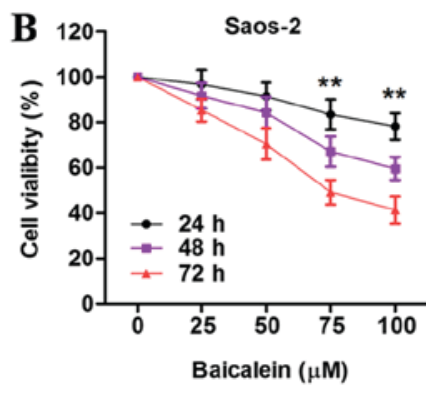

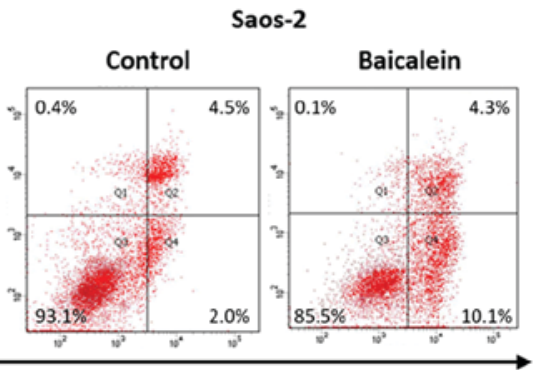

Annexin V-FITC
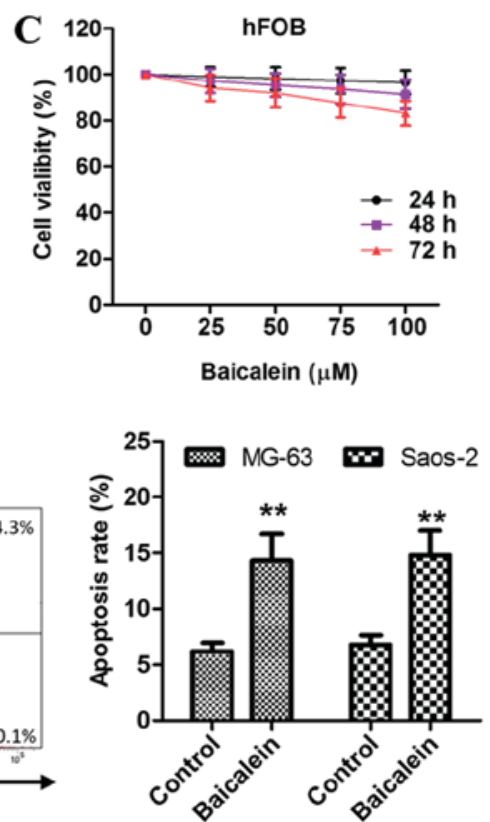

Figure 1. Baicalein regulates the proliferation and apoptosis of OS cells. (A-C) after exposure to various concentrations $(0,25,50,75,100 \mu \mathrm{M})$ of baicalein for 24, 48, $72 \mathrm{~h}$, the viability of MG-63, Saos-2 and hFOB cells was performed using the CCK-8 assay kit. DMSO was regarded as control. (D) MG-63 and Saos-2 cells were treated with $100 \mu \mathrm{M}$ baicalein for $48 \mathrm{~h}$, and then subjected to double staining with Annexin V-FITC and PI for flow cytometry detection. Q2+Q4 represents apoptotic cells (\%). Data is presented as the means \pm SD from three independent experiments. ${ }^{* * *} \mathrm{P}<0.01$ vs. control. OS, osteosarcoma; CCK-8, Cell Counting kit-8; DMSO, dimethyl sulphoxide; FITC, fluorescein isothiocyanate; SD, standard deviation.

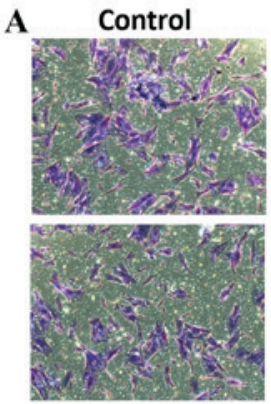

B

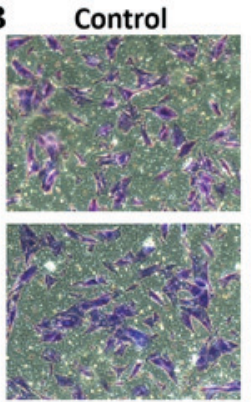

Baicalein

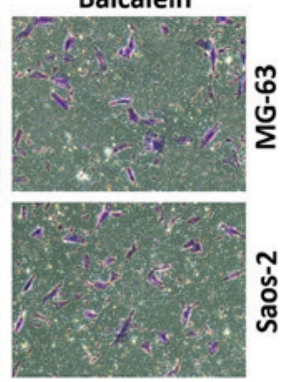

Baicalein

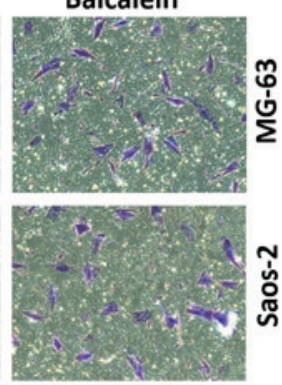

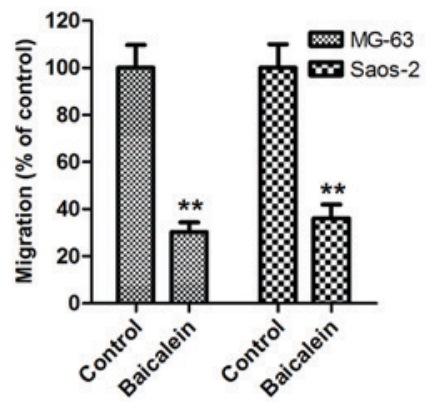

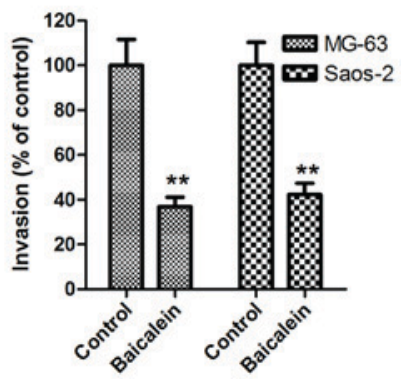

Figure 2. Cell invasion and migration assay showed that baicalein inhibited migration and invasion of MG-63 and Saos-2 cells. Following incubation for $48 \mathrm{~h}$ with $100 \mu \mathrm{M}$ baicalein, the MG-63 and Saos-2 cells on the lower surface of the Transwell insert were stained with crystal violet. Untreated cells (DMSO) were used as the controls. (A) The number of migratory MG-63 and Saos-2 cells was gradually decreased compared with control group. (B) The invasion assay was carried out in the same manner as the migration assay within the above cells. Representative images of migrated and invaded cells on the membrane (magnification, $\mathrm{x} 200$ ). Data are the mean $\pm \mathrm{SD}$ from three independent experiments. ${ }^{* *} \mathrm{P}<0.01$ vs. control. DMSO, dimethyl sulphoxide; SD, standard deviation.

luciferase reporter gene with 3'-UTR of Ezrin downstream (WT-Ezrin), its mutant version (MUT-Ezrin) via the binding site mutagenesis were also constructed (Fig. 3C). As shown in Fig. 3D, the luciferase activity of cells transfected with miR-183 mimic was dramatically decreased in wild type, but not mutant. Furthermore, the RT-qPCR and western blot analyses showed that overexpression of miR-183 noteworthily reduced the levels of Ezrin mRNA and protein compared with control group, whereas an opposite effect appeared in response to miR-183 inhibitor (Fig. 3E and F). Overall, these results demonstrate that miR-183 directly targets the 3'-UTR of Ezrin and negatively regulates its expression. 
$\mathbf{A}$

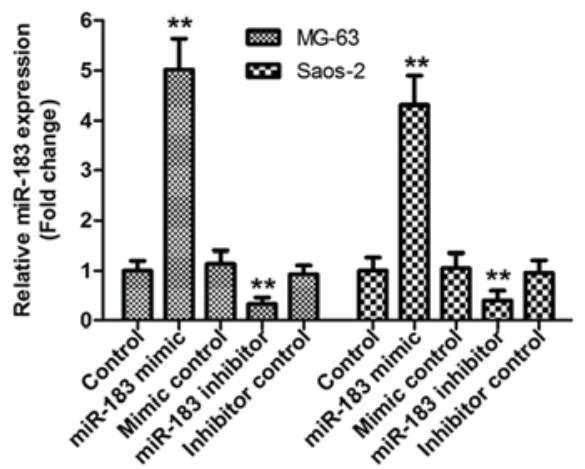

B

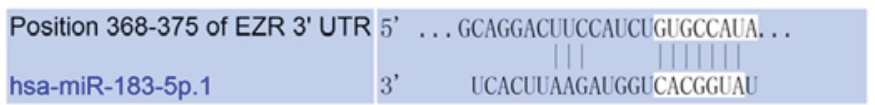

\section{CMV promoter $\quad$ Luciferase $\quad$ Ezrin 3'UTR (position 368-375)

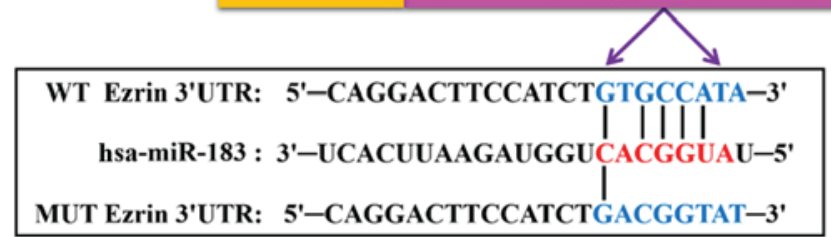

D

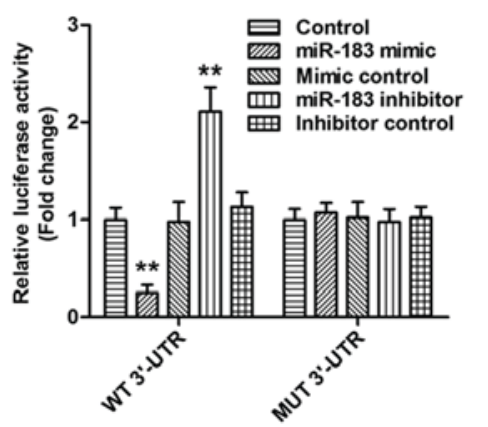

$\mathbf{E}$

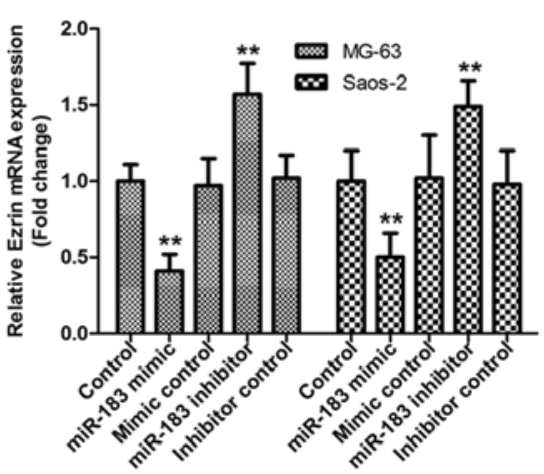

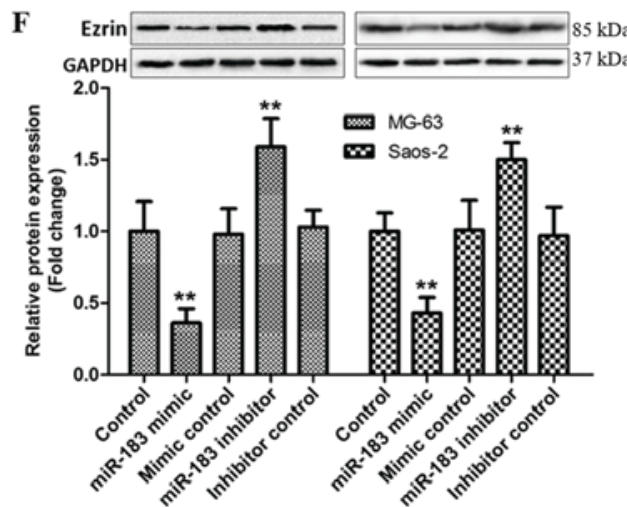

Figure 3. Ezrin was a direct target gene of miR-183. (A) After transfection of MG-63 or Saos-2 cells with $20 \mathrm{nM}$ miR-183 mimic/negative controls and $40 \mathrm{nM}$ miR-183 inhibitors/negative controls for $24 \mathrm{~h}$, the miR-183 levels were measured by RT-qPCR. (B) Base pairing complement provided by TargetScan suggested that the 3'-UTR of Ezrin was a potential target of miR-183. (C) The schematic representation of WT and MUT Ezrin 3'-UTR-pMIR-REPORT vector constructs. (D) Cells were co-transfected with $20 \mathrm{nM}$ miR-183 mimic/negative controls or $40 \mathrm{nM} \mathrm{miR-183} \mathrm{inhibitors/negative} \mathrm{controls} \mathrm{and} \mathrm{WT-pMIR-REPORT} \mathrm{or}$ MUT-pMIR-REPORT of Ezrin genes for $48 \mathrm{~h}$. The luciferase activity levels were measured using Dual-Luciferase Reporter Assay System. (E) and (F) After transfection of MG-63 or Saos-2 cells with $20 \mathrm{nM}$ miR-183 mimic/negative controls or $40 \mathrm{nM}$ miR-183 inhibitors/negative controls, the Ezrin mRNA and protein expression levels were determined RT-qPCR and western blot. The results are shown as the mean \pm SD of three independent experiments. ${ }^{* *} \mathrm{P}<0.01$ vs. control. WT, wild-type; MUT, mutant; miR, microRNA; RT-qPCR, reverse transcription-quantitative polymerase chain reaction; UTR, 3'-untranslated region; SD, standard deviation.

Baicalein increases miR-183 levels and represses Ezrin expression in OS cells. We firstly detected the contents of miR-183 and Ezrin in MG-63, Saos-2 and hFOB cells, respectively. RT-qPCR showed that miR-183 levels in MG-63 and Saos-2 cells were lower than those in hFOB cells (Fig. 4A). However, the expression of Ezrin mRNA and protein displayed an opposed tendency in above cells (Fig. 4A and B). These data indicate that abnormal expressions of miR-183 and Ezrin might be correlated highly with the pathogenesis and metastasis of OS.

Next, the underlying effects of baicalein on miR-183/Ezrin pathway were investigated using RT-qPCR and western blot in MG-63 and Saos-2 cells treated by $100 \mu \mathrm{M}$ baicalein for 48 h. As demonstrated in Fig. 4C, baicalein-stimulated MG-63 and Saos- 2 cells displayed a significant increase in miR-183 level in comparison with control group. However, the alterations in the mRNA and protein levels of Ezrin presented an opposite tendency in above parallel groups (Fig. 4D and E). In conclusion, these data corroborate that stimulation of baicalein is able to promote the generation of miR-183 and then retard the expression of Ezrin in MG-63 and Saos-2 cells.

miR-183/Ezrin pathway is required for the effects of baicalein on OS cells. As evidenced above experiments, baicalein represses the proliferation and migration and invision abilitiess, and participates in the activation of apoptosis in MG-63 and Saos-2 cells. Meanwhile, it as well negatively regulates the Ezrin expression via upregulating miR-183 level in MG-63 and Saos-2 cells. Therefore, we hypothesized that the miR-183/Ezrin pathway might be responsible for the antitumor effects of baicalein on OS cells. To verify the assumption, MG-63 and Saos-2 cells were co-cultured with baicalein and miR-183 inhibitors or pcDNA3.1-Ezrin, which showed a higher transfection efficiency (Fig. 5A). As expected, miR-183 inhibitor significantly reversed baicalein-induced high apoptosis rate and low proliferation, migration and invasive ability in MG-63 and Saos-2 cells, compared with baicalein group (Fig. 5B-E). Besides, we also found that overexpression of Ezrin by pcDNA3.1-Ezrin exerts similary effect in stumilation of baicalein with MG-63 and Saos-2 cells (Fig. 5B-E). Taken together, these observations indicate that baicalein induces apoptosis and attenuates proliferation and migration and invasion abilities of MG-63 and Saos-2 cells via activating miR-183/Ezrin pathway.

\section{Discussion}

OS is originated from mesenchymal stem cells producing osteoid or immature bone, and mainly characterized by high aggressiveness and metastasis. Multi-drug chemotherapy approaches for OS patients have obviously improved the 
$\mathbf{A}$
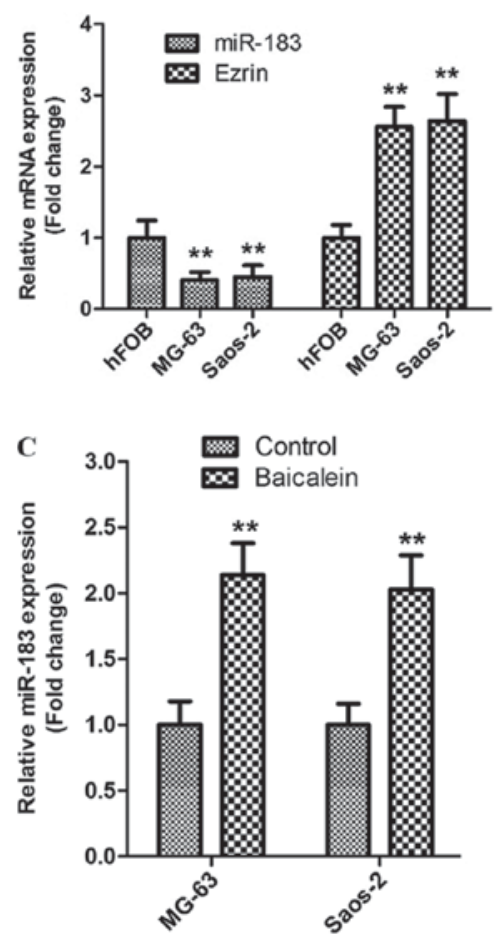

B
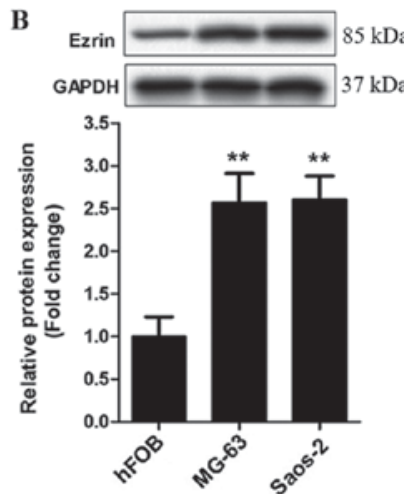

D

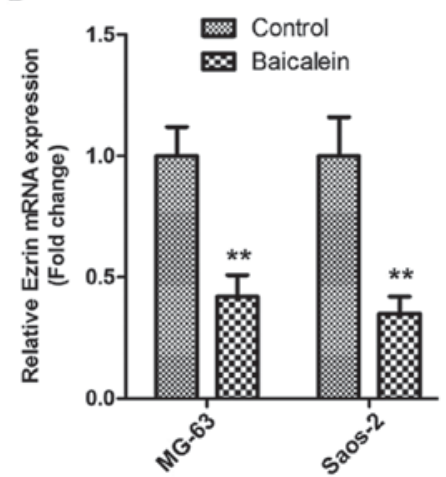

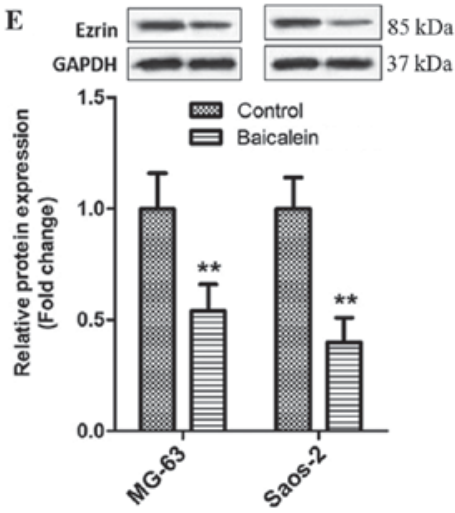

Figure 4. Roles of baicalein in miR-183 and Ezrin expression in MG-63 and Saos-2 cells. (A and B) In the MG-63, Saos-2 and hFOB cells, the mRNA levels of miR-183 and Ezrin were dectected by RT-qPCR, the protein levels of Ezrin were measured by western blot. The MG-63 or Saos-2 cells were subjected to $100 \mu \mathrm{M}$ baicalein for $48 \mathrm{~h}$. (C and D) The levels of miR-183 and Ezrin mRNA were detected by RT-qPCR, respectively. (E) Western blot assay was used to estimate the product of Ezrin protein. The results are shown as the mean $\pm \mathrm{SD}$ of three independent experiments. ${ }^{* * *} \mathrm{P}<0.01 \mathrm{vs}$. control. RT-qPCR, reverse transcription-quantitative polymerase chain reaction; miR, microRNA; SD, standard deviation.

survival rate subsequent to surgical resection (19). However, it is undesirable to the drug-resistance and life-threatening side effects induced by chemotherapy, leading to treatment failure or shorter survival. Thus, there is an urgent need for newer effective and low-toxic agents for patients with OS, especially for patients suffered from metastatic disease or disease recurrence. Enhancing the understanding of basic biology of therapeutic agents to OS has been a high priority in recent years.

Baicalein, a bioactive flavone purified from SB, is widely used in nutraceuticals and pharmaceuticals. It has been shown that baicalein has considerable potential in the treatment and prevention of cancers by inducing apoptosis, inhibiting the colony formation, migration and invasion $(3,20)$. Moreover, baicalein only suppresses the growth of cancer cells without the effects on normal cells $(21,22)$. In addition, researches have shown that baicalein can inhibit the progression of OS cells by a variety of biological pathways. Baicalein suppresses progression of OS cells via inactivating the Wnt/ $\beta$-catenin signaling pathway (23). Zhang and his colleagues reported that baicalein inhibitors reduces the viability of MG-63 cells by repressing c-MYC expression through the Wnt signaling pathway (24). Baicalein induces MG-63 cells apoptosis via ROS-induced BNIP3 expression (25). In the present study, we found that baicalein markedly promoted apoptosis and inhibited cell proliferation, migration and invasion of MG-63 and Saos-2 cells, but not hFOB cells. Taken together, these studies suggest that baicalein may be a promising and potential candidate for chemotherapeutic agents to treat OS. However, the precision mechanism by which baicalein mediates the development and progression of OS cells remains unclear.

Ectopic expression of miRNAs has been shown to be implicated in regulating proliferation, apoptosis, migration and invasion in multifarious cancer cells, including OS cells (26-28). These small molecules have emerged as potential targets for the diagnosis, therapy and prognosis of OS. MiR-183 has been identified as a promising biomarker, which was attributed to its early cancer detection and exact prognosis as well as high-efficiency treatment (29). Previous reports have shown that downexpression of miR-183 was observed in OS tissues or various cell lines $(15,30,31)$. Similarly, our previous research and this current study also revealed a similar tendency for miR-183 expression in OS MG-63 and Saos-2 cells (16). These data suggest that downregulation of miR-183 plays a pivotal role in promoting aggressiveness of OS.

It is generally known that the effect of miRNAs on the target gene is achieved by binding to the 3'-UTR of target gene. Ezrin, a target gene of miR-183, is a widely explored oncogene in various cancers, and as well involved in the tumorgenesis of OS. Our previous data and present research have demonstrated that Ezrin expression was markedly increased in MG-63 and Saos- 2 cells, promoting invasion and migration of cells; by contrast, these effects were abolished by knockdown of Ezrin (16). Furthermore, positive Ezrin expression is closely correlated with clinical grade, free-metastasis or metastasis, and adverse prognosis of patients with OS $(32,33)$. Thereby, 

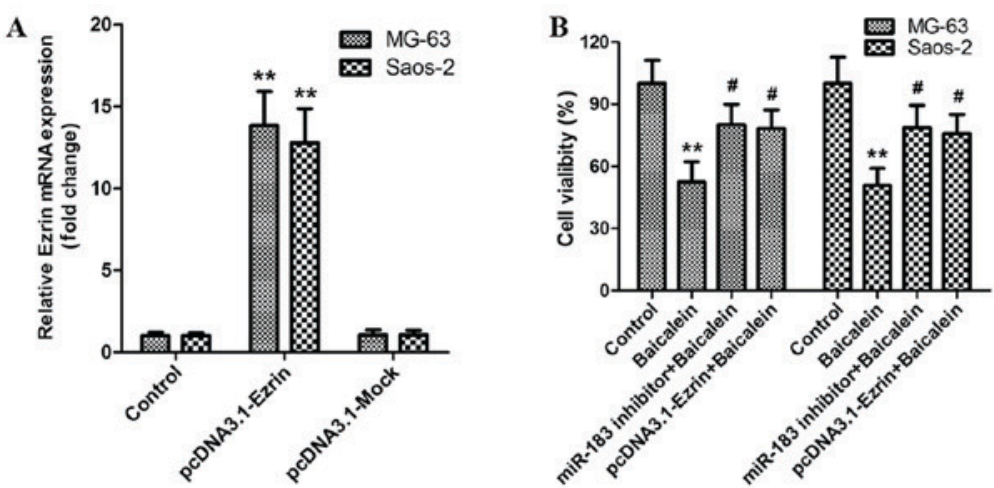

C
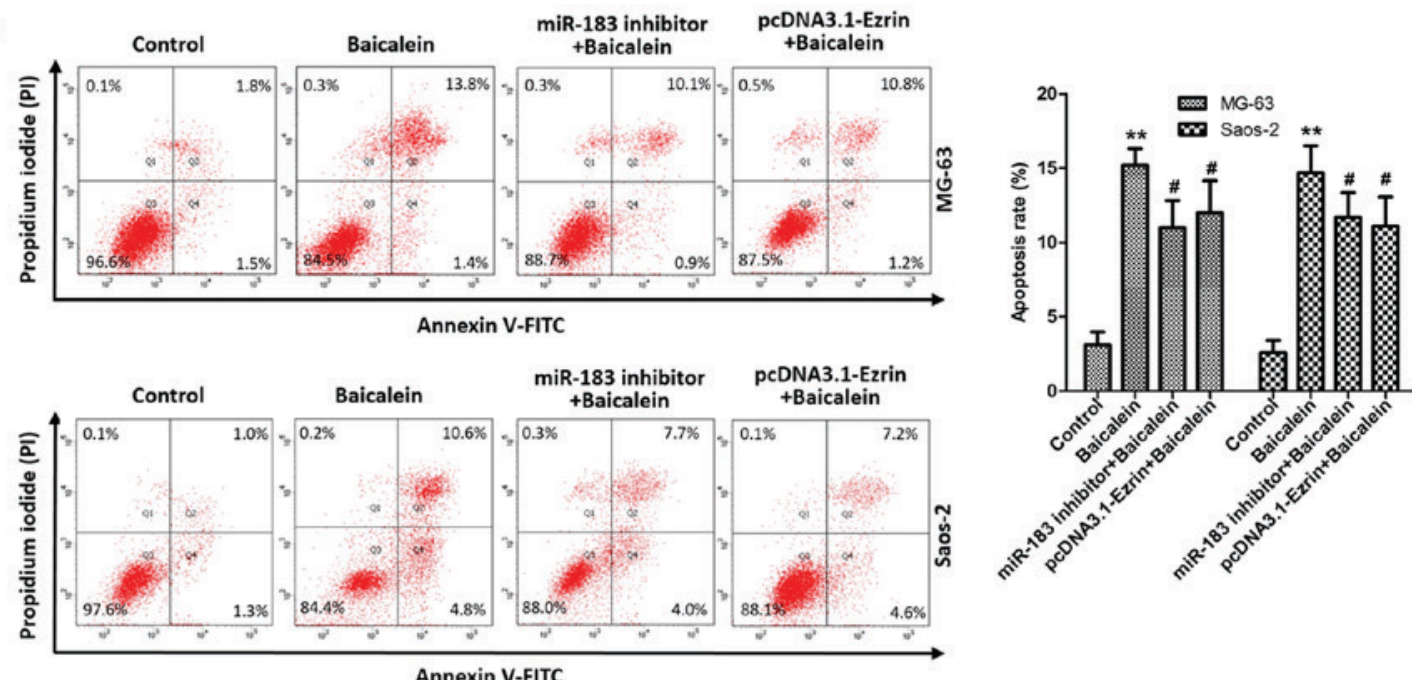

D
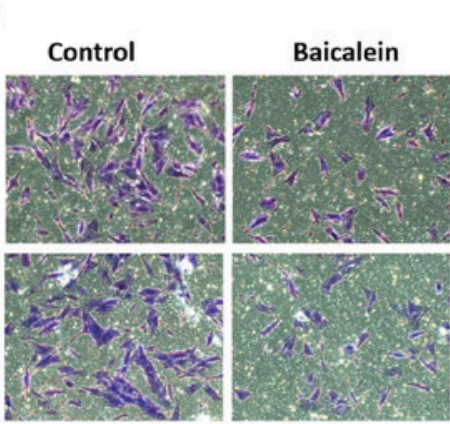

miR-183 inhibitor pcDNA3.1-Ezrin+ + Baicalein

Baicalein+

$\mathbf{E}$
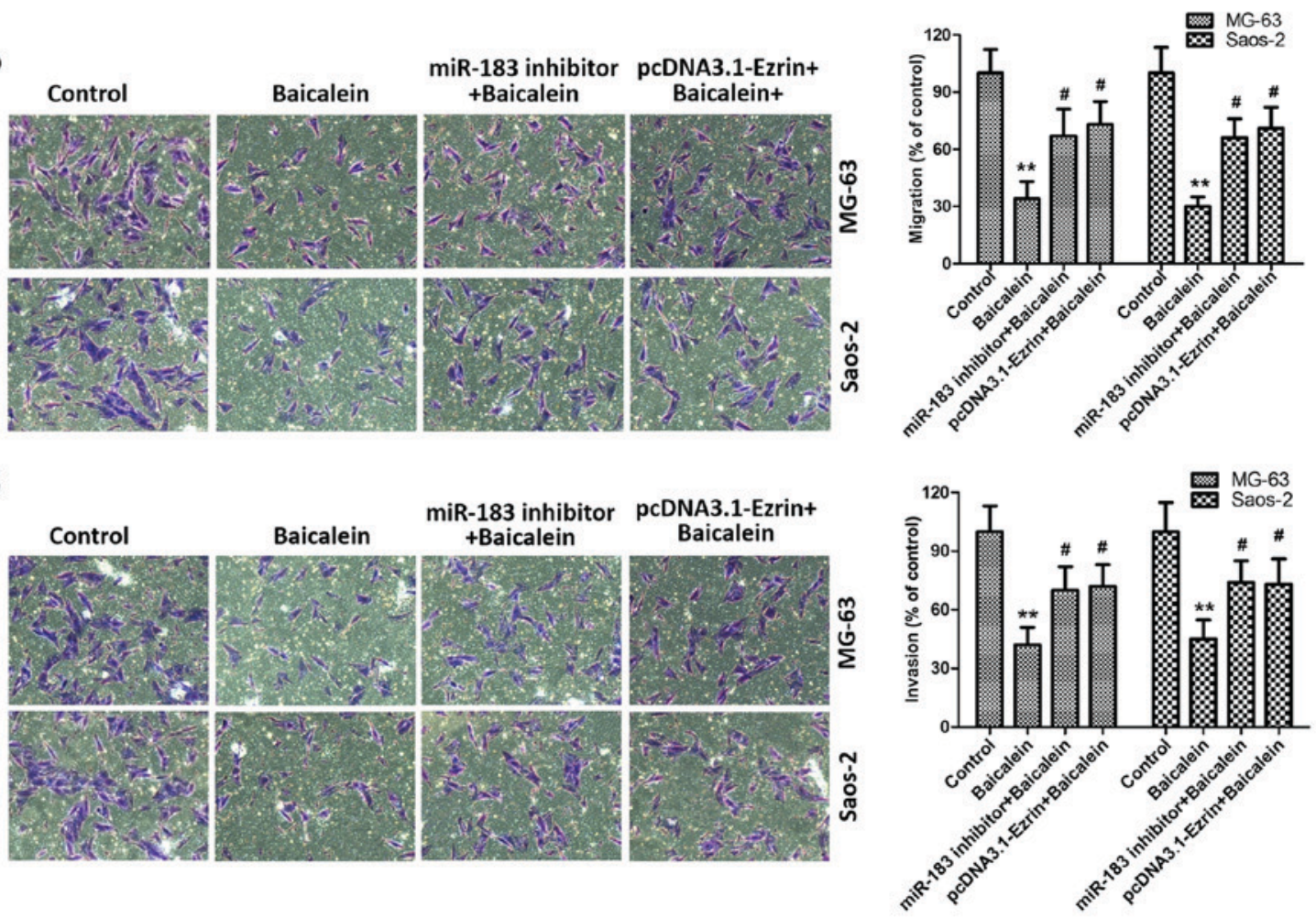

Figure 5. Baicalein exhibits antitumor effects by miR-183/Ezrin pathway in MG-63 and Saos-2 cells. MG-63 and Saos-2 cells were co-cultured with baicalein and miR-183 inhibitors or pcDNA3.1-Ezrin. Untreated cells (DMSO) were used as the controls. (A) The levels of Ezrin mRNA were detected using RT-qPCR to measure transfection efficiency. (B) Cell viability was estimated using the CCK-8 assay kit. (C) The double staining with Annexin V-FITC and PI was conducted by flow cytometry detection. Q2+Q4 represents apoptotic cells (\%). (D) and (E) The number of migratory and invasive MG-63 or Saos-2 cells were respectively determined by cell invasion and migration assay. Representative images of migrated and invaded cells on the membrane (magnification, $\mathrm{x} 200$ ). Data are the mean $\pm \mathrm{SD}$ from three independent experiments. ${ }^{* *} \mathrm{P}<0.01$ vs. control group, ${ }^{*} \mathrm{P}<0.01$ vs. baicalein group. RT-qPCR, reverse transcription-quantitative polymerase chain reaction; miR, microRNA; PI, propidium iodide; DMSO, dimethyl sulphoxide; CCK-8, Cell Counting kit-8; FITC, fluorescein isothiocyanate; SD, standard deviation. 
Ezrin may serve as a contributor for the invasiveness and carcinogenesis of OS.

Increasing evidence has illustrated that the miR-183/Ezrin pathway is involved in the progression of OS. It has been shown that downexpression of miR-183 promotes migration and invasion of OS by targeting Ezrin (15). MiR-183 inhibits the metastasis of OS via downregulation of Ezrin expression in F5M2 cells (31). Interestingly, research has shown that the aberrant expression of miR-183/Ezrin axis may be related to the prediction of aggressiveness and poor prognosis for patients with OS (30). In the current study, we validated that Ezrin was a target gene of miR-183, suggesting that miR-183 negatively regulated Ezrin expression. Given the roles of baicalein or miR-183/Ezrin pathway in OS, we rationally hypothesize that the antitumor effects of baicalein on OS were presented by activating the miR-183/Ezrin pathway. In this study, we found that baicalein significantly increased the miR-183 levels and decreased Ezrin expression in human OS MG-63 and Saos-2 cells. In addition, our findings showed that transfection of miR-183 inhibitor or overexpression of Ezrin abolished baicalein-induced antitumor effects in MG-63 and Saos- 2 cells. Thus, the miR-183/Ezrin pathway is involved in the suppressive effects of baicalein on MG-63 and Saos-2 cells.

In summary, our study clearly demonstrates that baicalein enhances apoptosis and inhibits cell proliferation, migration and invasion by activation of miR-183/Ezrin pathway in human OS MG-63 and Saos-2 cells. These findings reveal a molecular mechanism by which baicalein inhibits progression of OS, providing in vitro evidence to support baicalein as an efficient agent for the treatment of OS.

\section{Acknowledgements}

The authors would like to thank all of the volunteers who took part in this study.

\section{Funding}

Financial support of this study was obtained from the Natural Science Foundation in Hunan Province, China (grant no. 2015JJ4043), the Key Project of Health and Family Planning Commission in Hunan Province (grant no. A2017016), Clinical Research Center For Spinal Minimally Invasive Techniques in Hunan Province (grant no. 2017SK4004) and the Key Research and Development Plan in Hunan Province (grant no. 2017SK2104).

\section{Availability of data and materials}

The datasets used and/or analyzed during the current study are available from the corresponding author on reasonable request.

\section{Authors' contributions}

JZ performed the molecular experiments and drafted the manuscript. WY, Y-BZ, Y-XX, L-SW and W-KH performed the statistical analysis. W-JW conceived and designed the current study and revised the manuscript. All authors read and approved the final manuscript.

\section{Ethics approval and consent to participate}

Not applicable.

\section{Consent for publication}

Not applicable.

\section{Competing interests}

The authors declare that they have no competing interests.

\section{References}

1. Li J, Yang Z, Li Y, Xia J, Li D, Li H, Ren M, Liao Y, Yu S, Chen Y, et al: Cell apoptosis, autophagy and necroptosis in osteosarcoma treatment. Oncotarget 7: 44763-44778, 2016.

2. Zhang J, Yan YG, Wang C, Zhang SJ, Yu XH and Wang WJ: MicroRNAs in osteosarcoma. Clin Chim Acta 444: 9-17, 2015.

3. Zhou RT, He M, Yu Z, Liang Y, Nie Y, Tai S and Teng CB: Baicalein inhibits pancreatic cancer cell proliferation and invasion via suppression of NEDD9 expression and its downstream Akt and ERK signaling pathways. Oncotarget 8: 56351-56363, 2017.

4. Liu X, Liu S, Chen J, He L, Meng X and Liu S: Baicalein suppresses the proliferation of acute T-lymphoblastic leukemia Jurkat cells by inhibiting the Wnt $/ \beta$-catenin signaling. Ann Hematol 95: 1787-1793, 2016.

5. Wei N, Wei Y, Li B and Pang L: Baicalein promotes neuronal and behavioral recovery after intracerebral hemorrhage via suppressing apoptosis, Oxidative stress and neuroinflammation. Neurochem Res 42: 1345-1353, 2017.

6. Ye H, Zhang Y, Wang Y, Xia J, Mao X and Yu X: The restraining effect of baicalein and U0126 on human cervical cancer cell line HeLa. Mol Med Rep 16: 957-963, 2017.

7. Chai Y, Xu J and Yan B: The anti-metastatic effect of baicalein on colorectal cancer. Oncol Rep 37: 2317-2323, 2017.

8. Choi EO, Park C, Hwang HJ, Hong SH, Kim GY, Cho EJ, Kim WJ and Choi YH: Baicalein induces apoptosis via ROS-dependent activation of caspases in human bladder cancer 5637 cells. Int J Oncol 49: 1009-1018, 2016.

9. Ma X, Yan W, Dai Z, Gao X, Ma Y, Xu Q, Jiang J and Zhang S: Baicalein suppresses metastasis of breast cancer cells by inhibiting EMT via downregulation of SATB1 and Wnt/ $\beta$-catenin pathway. Drug Des Devel Ther 10: 1419-1441, 2016.

10. Palmini G, Marini F and Brandi ML: What is new in the miRNA world regarding osteosarcoma and chondrosarcoma? Molecules 22: pii: E417, 2017.

11. Zoni E and van der Pluijm G: The role of microRNAs in bone metastasis. J Bone Oncol 5: 104-108, 2016.

12. Cao LL, Xie JW, Lin Y, Zheng CH, Li P, Wang JB, Lin JX, Lu J, Chen QY and Huang CM: miR-183 inhibits invasion of gastric cancer by targeting Ezrin. Int J Clin Exp Pathol 7: 5582-5594, 2014.

13. Li M, Feng YM and Fang SQ: Overexpression of ezrin and galectin-3 as predictors of poor prognosis of cervical cancer. Braz J Med Biol Res 50: e5356, 2017.

14. Kong J, Li Y, Liu S, Jin H, Shang Y, Quan C, Li Y and Lin Z: High expression of ezrin predicts poor prognosis in uterine cervical cancer. BMC Cancer 13: 520, 2013.

15. Zhu J, Feng Y, Ke Z, Yang Z, Zhou J, Huang X and Wang L: Down-regulation of miR-183 promotes migration and invasion of osteosarcoma by targeting Ezrin. Am J Pathol 180: 2440-2451, 2012.

16. Zhang J, Zuo J, Lei M, Wu S, Zang X and Zhang C: Ezrin promotes invasion and migration of the MG63 osteosarcoma cell. Chin Med J (Engl) 127: 1954-1959, 2014.

17. Livak KJ and Schmittgen TD: Analysis of relative gene expression data using real-time quantitative PCR and the 2(-Delta Delta C(T)) method. Methods 25: 402-408, 2001.

18. Kushlinskii NE, Fridman MV and Braga EA: Molecular mechanisms and microRNAs in osteosarcoma pathogenesis. Biochemistry (Mosc) 81: 315-328, 2016.

19. Anderson ME: Update on survival in osteosarcoma. Orthop Clin North Am 47: 283-292, 2016. 
20. Gao Y, Snyder SA, Smith JN and Chen YC: Anticancer properties of baicalein: A review. Med Chem Res 25: 1515-1523, 2016.

21. Dinda B, Dinda S, DasSharma S, Banik R, Chakraborty A and Dinda M: Therapeutic potentials of baicalin and its aglycone, baicalein against inflammatory disorders. Eur J Med Chem 131: 68-80, 2017.

22. Chung H, Choi HS, Seo EK, Kang DH and Oh ES: Baicalin and baicalein inhibit transforming growth factor- $\beta 1$-mediated epithelial-mesenchymal transition in human breast epithelial cells. Biochem Biophys Res Commun 458: 707-713, 2015.

23. Dai G, Zheng D, Wang Q, Yang J, Liu G, Song Q, Sun X, Tao C, $\mathrm{Hu} \mathrm{Q}$, Gao T, et al: Baicalein inhibits progression of osteosarcoma cells through inactivation of the Wnt/ $\beta$-catenin signaling pathway. Oncotarget 8: 86098-86116, 2017.

24. He N and Zhang Z: Baicalein suppresses the viability of MG-63 osteosarcoma cells through inhibiting c-MYC expression via Wnt signaling pathway. Mol Cell Biochem 405: 187-196, 2015.

25. Ye F, Wang H, Zhang L, Zou Y, Han H and Huang J: Baicalein induces human osteosarcoma cell line MG-63 apoptosis via ROS-induced BNIP3 expression. Tumour Biol 36: 4731-4740, 2015.

26. Pal MK, Jaiswar SP, Dwivedi VN, Tripathi AK, Dwivedi A and Sankhwar P: MicroRNA: A new and promising potential biomarker for diagnosis and prognosis of ovarian cancer. Cancer Biol Med 12: 328-341, 2015.
27. Taylor MA and Schiemann WP: Therapeutic opportunities for targeting microRNAs in cancer. Mol Cell Ther 2: 1-13, 2014.

28. Nugent M: microRNA and bone cancer. Adv Exp Med Biol 889: 201-230, 2015.

29. Zhang QH, Sun HM, Zheng RZ, Li YC, Zhang Q, Cheng P, Tang ZH and Huang F: Meta-analysis of microRNA-183 family expression in human cancer studies comparing cancer tissues with noncancerous tissues. Gene 527: 26-32, 2013

30. Mu Y, Zhang $\mathrm{H}$, Che L and Li K: Clinical significance of microRNA-183/Ezrin axis in judging the prognosis of patients with osteosarcoma. Med Oncol 31: 821, 2014.

31. Zhao H, Guo M, Zhao G, Ma Q, Ma B, Qiu X and Fan Q: miR-183 inhibits the metastasis of osteosarcoma via downregulation of the expression of Ezrin in F5M2 cells. Int J Mol Med 30: 1013-1020, 2012.

32. Abdou AG, Kandil M, Asaad NY, Dawoud MM, Shahin AA and Abd Eldayem AF: The prognostic role of Ezrin and HER2/neu expression in osteosarcoma. Appl Immunohistochem Mol Morphol 24: 355-363, 2016.

33. Zhao DH, Zhu J, Wang WB, Dong F, Zhang Q, Fan HW Zhang JZ and Wang YM: Correlations of ezrin expression with pathological characteristics and prognosis of osteosarcoma: A meta-analysis. ScientificWorldJournal 2014: 837543, 2014. 